\title{
Tightening the belt: Constraining the mass and evolution in SDC335*
}

\author{
A. Avison ${ }^{1,2}$, N. Peretto ${ }^{3}$, G. A. Fuller ${ }^{1,2}$, A. Duarte-Cabral ${ }^{4}$, A. Traficante ${ }^{1}$, and J. E. Pineda ${ }^{5}$ \\ 1 Jodrell Bank Centre for Astrophysics, School of Physics and Astronomy, University of Manchester, Manchester, M13 9PL, UK \\ e-mail: adam.avison@manchester.ac .uk \\ 2 UK ALMA Regional Centre Node \\ 3 School of Physics and Astronomy, Cardiff University, Queens Buildings, The Parade, Cardiff, CF24 3AA, UK \\ 4 School of Physics and Astronomy, University of Exeter, Stocker Road, Exeter, EX4 4QL, UK \\ 5 ETH Zürich, Institut für Astronomie, Wolfgang-Pauli-Str. 27, 8093 Zürich, Switzerland
}

Received 23 September 2014 / Accepted 15 January 2015

\begin{abstract}
Aims. Recent ALMA observations identified one of the most massive star-forming cores yet observed in the Milky Way: SDC335MM1, within the infrared dark cloud SDC335.579-0.292. Along with an accompanying core MM2, SDC335 appears to be in the early stages of its star formation process. We aim to constrain the properties of the stars forming within these two massive millimetre sources.

Methods. Observations of SDC335 at 6,8,23 and $25 \mathrm{GHz}$ were made with the Australia Telescope Compact Array. We report the results of these continuum measurements, which combined with archival data, allow us to build and analyse the spectral energy distributions (SEDs) of the compact sources in SDC335.

Results. Three hyper-compact HII regions within SDC335 are identified, two of which are within the MM1 core. For each HCHII region, we fit a free-free emission curve to the data, providing the derivation of the sources' emission measure, ionising photon flux, and electron density. Using these physical properties we assign each HCHII region a zero-age main sequence (ZAMS) spectral type, finding two protostars with characteristics of spectral type B1.5 and one with a lower limit of B1-B1.5. Ancillary data from infrared to $\mathrm{mm}$ wavelength are used to construct free-free component subtracted SEDs for the mm-cores, which allows us to calculate the bolometric luminosities and revise the previous gas mass estimates.

Conclusions. The measured luminosities for the two mm-cores are lower than expected from accreting sources displaying characteristics of the ZAMS spectral type assigned to them. The protostars are still actively accreting, suggesting that a mechanism is limiting the accretion luminosity. We present the case for two different mechanisms capable of causing lower than expected accretion luminosity. Finally, using the ZAMS mass values as lower limit constraints, a final stellar population for SDC335 was synthesised finding SDC335 is likely to be in the process of forming a stellar cluster comparable to the Trapezium cluster and NGC 6334 I(N).
\end{abstract}

Key words. stars: formation - ISM: clouds - HII regions - stars: massive - stars: protostars

\section{Introduction}

The massive infrared dark cloud (IRDC) SDC335.579-0.292 (Peretto \& Fuller 2009) has been noted as an object of interest because of its high mass, but low levels of radiative feedback for the protostars inhabiting it. The lack of the disruptive effects of radiative feedback allow SDC335 to be used to observe the initial conditions of star formation.

SDC335 shows an interesting filamentary structure (Fig. 1), with six filamentary arms radiating outward from a denser central region. Within this central region reside two infrared bright cores, MM1 and MM2. The cores are separated by 20.2" equivalent to $0.32 \mathrm{pc}$ at the distance of SDC335 which throughout this work is taken as $3.25\left(_{-0.35}^{+0.33}\right) \mathrm{kpc}$ (following Peretto et al. 2013, using the Reid et al. 2009 model).

Both cores are associated with $6.7 \mathrm{GHz}$ class II methanol masers (Caswell et al. 2011), with two methanol masers associated with MM1 and a single maser associated with MM2. The

* FITS images of the ATCA data at $6,8,23$, and $25 \mathrm{GHz}$ used in this paper are only available at the CDS via anonymous ftp to cdsarc.u-strasbg.fr (130.79.128.5) or via http://cdsarc.u-strasbg.fr/viz-bin/qcat?J/A+A/577/A30 class II methanol maser is a unique tracer of massive star formation (Minier et al. 2003; Xu et al. 2008; Breen et al. 2013), meaning that SDC335 is harbouring massive protostellar objects. However, prior to this work no free-free emission at $6 \mathrm{~cm}$ had been detected to a sensitivity limit of $0.2 \mathrm{mJy}$ (Garay et al. 2002), indicating that these sources are indeed young and in a pre-ionising phase of their evolution.

SDC335 was the target of ALMA Cycle 0 observations with ALMA Band 3 (Peretto et al. 2013). ALMA observed the $3.2 \mathrm{~mm}$ dust continuum as well as spectral line transitions for $\mathrm{CH}_{3} \mathrm{OH}(13-12)$ and $\mathrm{N}_{2} \mathrm{H}^{+}(1-0)$. Using these data, it was shown that MM1 has a mass of $\sim 500 M_{\odot}$ making it one of the most massive star forming cores observed in the Galaxy. MM2 was found to have a mass of $\sim 50 M_{\odot}{ }^{1}$. The ALMA data supplemented by Spitzer, Herschel and Mopra data also shows that the whole of SDC335 was undergoing global collapse, and that the converging network of filaments feed the central region of the cloud with pristine gas.

\footnotetext{
We here state approximate masses for MM1 and MM2 as a calculation error has been found in Peretto et al. (2013) since publication, leading to $\sim 16 \%$ lower masses. This issue will be addressed in a forthcoming corrigendum.
} 


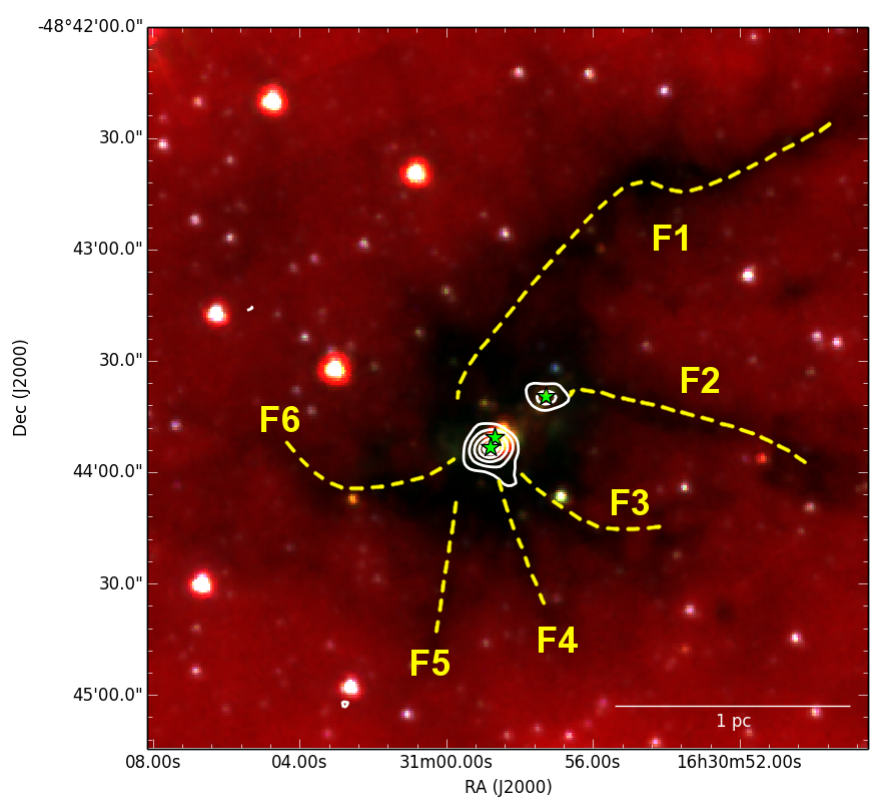

Fig. 1. Spitzer three colour image of the IRDC SDC335. Colour scale: red, green and blue GLIMPSE images at 8.0, 4.5 and $3.6 \mu \mathrm{m}$ respectively. White contours: ALMA $3.2 \mathrm{~mm}$ continuum, with contours at 3 , $10,25,50,75$ and $90 \%$ of the peak flux. Green $\star$, Class II $\mathrm{CH}_{3} \mathrm{OH}$ masers. The yellow dashed lines outline the filaments of SDC335, following Peretto et al. (2013). (Colour online.)

In this paper, we present follow-up observations of SDC335 made using the Australia Telescope Compact Array (ATCA) in the radio $(6,8,23$ and $25 \mathrm{GHz})$ and use these new data to better constrain the masses of the stars forming in MM1 and MM2. In addition, we discuss the implications of the presence of three newly identified HCHII regions associated with these millimetre sources.

\section{Observations}

\subsection{ATCA}

The primary source of data for this work is the ATCA, from two dedicated observing runs under project code C2835.

We made centimetre wavelength $(6$ and $8 \mathrm{GHz})$ observations using the ATCA over 13 hours on 9th/10th June 2013. During these observations the ATCA was in the " $6 \mathrm{C}$ " array configuration, giving a $u v$-range from $\sim 2$ to $140 \mathrm{k} \lambda$. These observations consisted purely of continuum observations with the CABB system (Wilson et al. 2011) set up to observe a $2 \mathrm{GHz}$ bandwidth centred simultaneously at both 6 and 8 GHz. We used PKS1934638 for flux and bandpass calibration and PKS1646-50 for phase calibration.

We took millimetre wavelength (23 and $25 \mathrm{GHz}$ ) observations in a single 11 hours session on the 13th September 2013, with the ATCA in the " $1.5 \mathrm{~A}$ " array configuration. Data from the longest ATCA baseline (all antenna 6 baselines) was flagged out to allow for better matching of $u v$-range to the 6 and $8 \mathrm{GHz}$ data during processing; the millimetre wavelength data therefore have a $u v$-range from $\sim 9$ to $127 \mathrm{k} \lambda$. We configured the CABB system to observe two $2 \mathrm{GHz}$ continuum bands centred at 23 and $25 \mathrm{GHz}$ plus seven "zoom" bands to cover six ammonia transitions and the water maser at $22 \mathrm{GHz}$. We only discuss the continuum data here, the ammonia and water maser data will appear in a later work. The flux and phase calibrators were the same as the
Table 1. Observing characteristics of interferometric studies of SDC335 used in this study.

\begin{tabular}{crcc}
\hline \hline Telescope & $\begin{array}{r}\text { Freq. } \\
{[\mathrm{GHz}]}\end{array}$ & $\begin{array}{c}\text { Continuum } \\
\text { Sensitivity } \\
{\left[\mathrm{mJy} \mathrm{bm}^{-1}\right]}\end{array}$ & $\begin{array}{c}\text { Synthesised } \\
\text { Beam } \\
{\left[{ }^{\prime \prime} \times{ }^{\prime \prime}\right]}\end{array}$ \\
\hline ALMA & 93.7 & 0.400 & $5.6 \times 4.0$ \\
ATCA & 25.0 & 0.023 & $1.9 \times 1.0$ \\
ATCA & 23.0 & 0.026 & $2.1 \times 1.1$ \\
ATCA & 8.0 & 0.008 & $2.3 \times 1.0$ \\
ATCA & 6.0 & 0.013 & $2.9 \times 1.4$ \\
\hline
\end{tabular}

6 and 8 GHz observations with PKS 1253-055 used for bandpass calibration.

These ATCA data were reduced using the data reduction package MIRIAD with standard ATNF reduction strategies. The task MFCLEAN ${ }^{2}$ was used to CLEAN the image owing to the large bandwidths of the data. We refer the reader to the MIRIAD user guide $^{3}$ for more information.

The beam and sensitivity characteristics of these observations are listed in Table 1, including the Peretto et al. (2013) ALMA observations of this object for comparison. We obtained integrated flux density measurements from these observations by fitting Gaussians to each source using the MIRIAD IMFIT task, with the exception of MM1 $a$ at $6 \mathrm{GHz}$ where the slight blending of the two MM1 sources led to unreliable gaussian fits, instead values for this source were measured "by hand" using a defined polygonal area in CASA (McMullin et al. 2007), leading to the increased uncertainty in the $6 \mathrm{GHz}$ integrated flux density value. The integrated flux density values are shown in Table 2.

\subsection{Additional data}

In addition to the ATCA observations listed above, data for SDC335 was available at $7 \mathrm{~mm}$ from ATCA (Avison, Cunningham \& Fuller, in prep., we note only MM1 is detected in these data) and ALMA at $3.2 \mathrm{~mm}$ (Peretto et al. 2013). We have also used archival data from Spitzer ${ }^{4}$ (MIPS and GLIMPSE), MSX, and APEX (LABOCA from the ATLASGAL survey; Schuller et al. 2009).

We also made use of Herschel data from the Herschel infrared Galactic Plane Survey (Hi-GAL; Molinari et al. 2010), which has mapped the whole Galactic Plane covering the wavelength range $70 \leq \lambda \leq 500 \mu \mathrm{m}$ using both the PACS (Poglitsch et al. 2010) and SPIRE (Griffin et al. 2010) photometry instruments in parallel mode on board of the Herschel Space Observatory (Pilbratt et al. 2010). We limited our analysis to the PACS 70 and $160 \mu \mathrm{m}$ data, which have the highest spatial resolution $\left(\simeq 5^{\prime \prime}\right.$ and $\simeq 12^{\prime \prime}$, respectively).

The source extraction and photometry of the Herschel data has been carried out with Hyper, an enhanced aperture photometry code specifically designed to account for high background variability and source crowding of the Galactic Plane data (Traficante et al. 2015). The background is evaluated locally for each source assuming different polynomial orders (from zero to the fifth order) to model the background fluctuations. The polynomial background-subtracted map with the lowest root

\footnotetext{
2 http://www.atnf.csiro.au/computing/software/miriad/ doc/mfclean.html

3 http://www.atnf.csiro.au/computing/software/miriad/ userguide/

4 Data from Spitzer is shown within Figs. 4 and 5 for illustrative purposes.
} 
Table 2. Observed results of the SDC335 millimetre and protostellar cores used in this study.

\begin{tabular}{cccccccc}
\hline \hline Instrument & $\begin{array}{c}\text { Freq. } \\
{[\mathrm{Hz}]}\end{array}$ & $\begin{array}{c}\lambda \\
{[\mathrm{m}]}\end{array}$ & $\begin{array}{c}\text { Resolution }^{\dagger} \\
{\left[{ }^{\prime \prime}\right]}\end{array}$ & & \multicolumn{3}{c}{$\begin{array}{c}S_{\text {int }} \\
{[\mathrm{Jy}]}\end{array}$} \\
\cline { 5 - 8 } & & & & MM1 & MM1 $a$ & MM1 $b$ & MM2 \\
\hline PACS & $4.28(12)$ & $70.0(-6)$ & 5.0 & $792 \pm 158$ & - & - & $604 \pm 121$ \\
PACS & $1.87(12)$ & $160.0(-6)$ & 12.0 & $1456 \pm 582.0$ & - & - & $961 \pm 384$ \\
LABOCA & $3.45(11)$ & $870.0(-6)$ & 18.0 & $8.92 \pm 5.34$ & - & - & $1.45 \pm 0.87$ \\
ALMA & $9.37(10)$ & $3.20(-3)$ & $5.6 \times 4.0$ & $1.01 \pm 0.10(-1)$ & - & - & $1.20 \pm 0.20(-2)$ \\
ATCA & $4.59(10)$ & $6.54(-3)$ & $3.2 \times 0.8$ & $6.85 \pm 1.76(-3)$ & & - & $<0.92(-4)^{\star}$ \\
ATCA & $4.32(10)$ & $6.95(-3)$ & $3.5 \times 0.9$ & $6.56 \pm 1.37(-3)$ & $3.14 \pm 0.91(-3)$ & - & $<0.89(-4)^{\star}$ \\
ATCA & $2.50(10)$ & $1.20(-2)$ & $1.9 \times 1.0$ & $2.21 \pm 0.32(-3)$ & $1.41 \pm 0.17(-3)$ & $7.12 \pm 0.12(-4)$ & $7.17 \pm 0.99(-4)$ \\
ATCA & $2.30(10)$ & $1.30(-2)$ & $2.1 \times 1.1$ & $2.48 \pm 0.35(-3)$ & $1.76 \pm 0.22(-3)$ & $6.02 \pm 0.12(-4)$ & $7.24 \pm 1.10(-4)$ \\
ATCA & $8.00(09)$ & $3.75(-2)$ & $2.3 \times 1.0$ & $6.07 \pm 0.98(-4)$ & $3.02 \pm 0.39(-4)$ & $3.00 \pm 0.36(-4)$ & $3.59 \pm 0.42(-4)$ \\
ATCA & $6.00(09)$ & $5.00(-2)$ & $2.9 \times 1.4$ & $3.33 \pm 0.52(-4)$ & $1.60 \pm 0.36(-4)$ & $2.25 \pm 0.25(-4)$ & $2.72 \pm 0.34(-4)$ \\
\hline
\end{tabular}

Notes. ${ }^{(\dagger)}$ For interferometers resolution given is the synthesised beam major and minor axes. $\left.{ }^{\star}\right) 3 \sigma$ upper limits on the none detection of MM2. Columns 2, 3 and 5-8 values in brackets are the order of magnitude in base 10 .

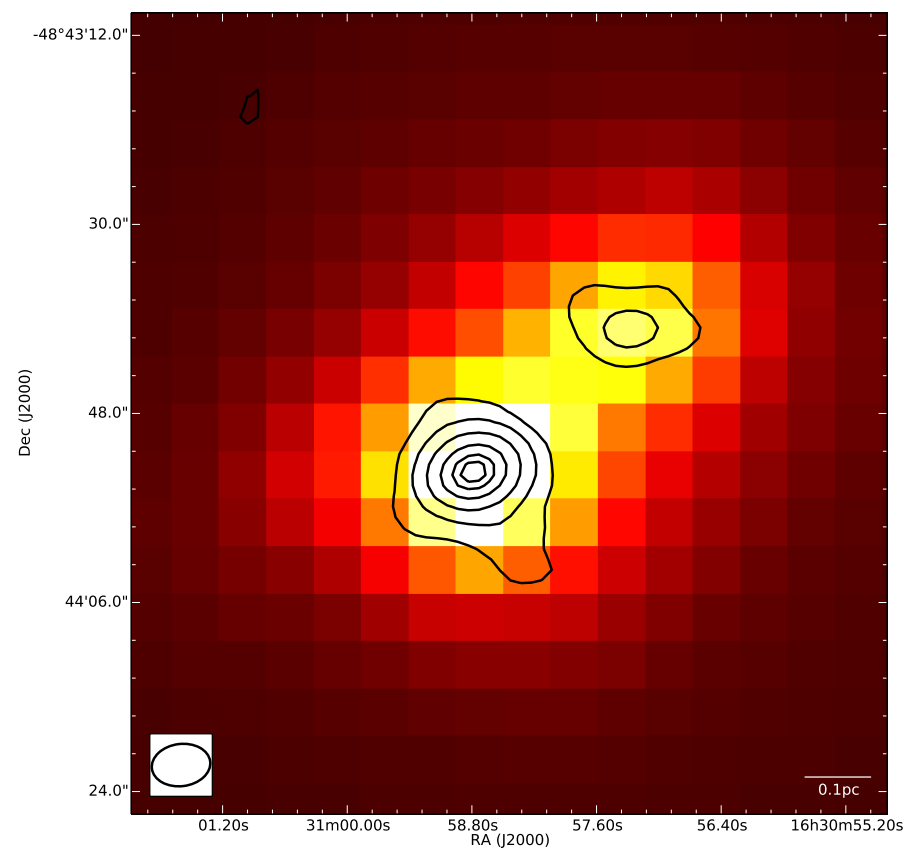

Fig. 2. PACS $160 \mu \mathrm{m}$ image of SDC335, overlaid with ALMA $3.2 \mathrm{~mm}$ data (contours). It is clear that MM1 and MM2 are blended in this image with emission from MM1 possibly affecting photometry for MM2. The ALMA synthesised beam shape is seen in the lower left inset. (Colour online.)

mean square is taken as reference to evaluate the source flux, and the equivalent polynomial background order as the best fit to describe the background fluctuations in the Hi-GAL maps.

In the case of overlapping sources, as for MM1 and MM2, the algorithm models all the sources with a multi-Gaussian fit and subtracts the model of the companions, prior to evaluating each source flux in the aperture region. Within SDC335, the proximity of the brighter MM1 core to MM2 at the resolution of Herschel means that the two cores are blended in both the 1.9 and $4.3 \mathrm{THz}(160$ and $70 \mu \mathrm{m})$ Herschel maps (see Fig. 2). The data points from these maps have the greatest influence on the SED fitting result (e.g. Fig. 5); we discuss this in Sect. 3.2. The flux estimation for each core can be affected by the flux of the overlapping companion or by a coarse background estimation. Although Hyper can estimate fluxes with a precision of a few percent even in complex background regions and in the presence of heavily blended sources (Traficante et al. 2015), we decided to be conservative and assume a flux uncertainty of $\pm 20 \%$ of the photometric Herschel flux values.

The source fluxes extracted with Hyper have been corrected for aperture corrections. The integrated fluxes from this extraction, $S_{\text {int }}$, are shown in Table 2.

\section{Results and analysis}

As viewed in the radio/mm of our observations, SDC335 is seen in Fig. 3. Comparing our ATCA observations with the ALMA data, we observe sources at the position of the two mm-cores, MM1 and MM2, in SDC335. However, given the increased resolution of our ATCA data we find that the MM1 core comprises two sources separated by $\sim 3^{\prime \prime}$. We name these MM1 $a$ and MM1 $b$, with MM1 $a$ being the more southerly of the pair (see Fig. 3b). The two fragments are situated 2.7" from one another, a physical separation of $\sim 9000$ AU at the distance of SDC335. MM2, the less massive mm-core in SDC335, remains a single source at this increased resolution. The position of each radio core is listed in Table 3.

The red and blue contours and arrows in Fig. 3 represent the $5 \%$ of peak emission boundary and direction of the HNC molecular outflow discovered in the ALMA Cycle 0 data (to be discussed in full in Pineda et al., in prep.), with the colours denoting red and blue shifted emission respectively. There is a slight kink in the red shifted outflow, and to indicate this we use two arrows for this feature. Of interest to our work is the fact that the position of maximal overlap between the two outflow arms coincides, to within $0.5^{\prime \prime}$, with the position of peak continuum flux from MM1a. As such we assume that MM1 $a$ is the source that drives the molecular outflow.

In Fig. 3 we also show the positions of three maser species: Class I (44 GHz) and Class II (6.7 GHz) $\mathrm{CH}_{3} \mathrm{OH}$ (with positions from Avison, Cunningham \& Fuller, in prep. and Caswell et al. 2011, respectively) and $22 \mathrm{GHz} \mathrm{H}_{2} \mathrm{O}$ (positions from Breen et al. 2010, and the $22 \mathrm{GHz}$ spectral data from our ATCA observations). Class II $\mathrm{CH}_{3} \mathrm{OH}$ and $\mathrm{H}_{2} \mathrm{O}$ masers are typically associated with the central regions of protostellar objects. Class II $\mathrm{CH}_{3} \mathrm{OH}$ masers are seen to trace discs or disc-like features when observed on milli-arcsecond scales (e.g. Bartkiewicz et al. 2009) and are uniquely associated with regions of massive star formation. $\mathrm{H}_{2} \mathrm{O}$ masers are typically associated with outflow regions in both high and low mass star-forming regions 

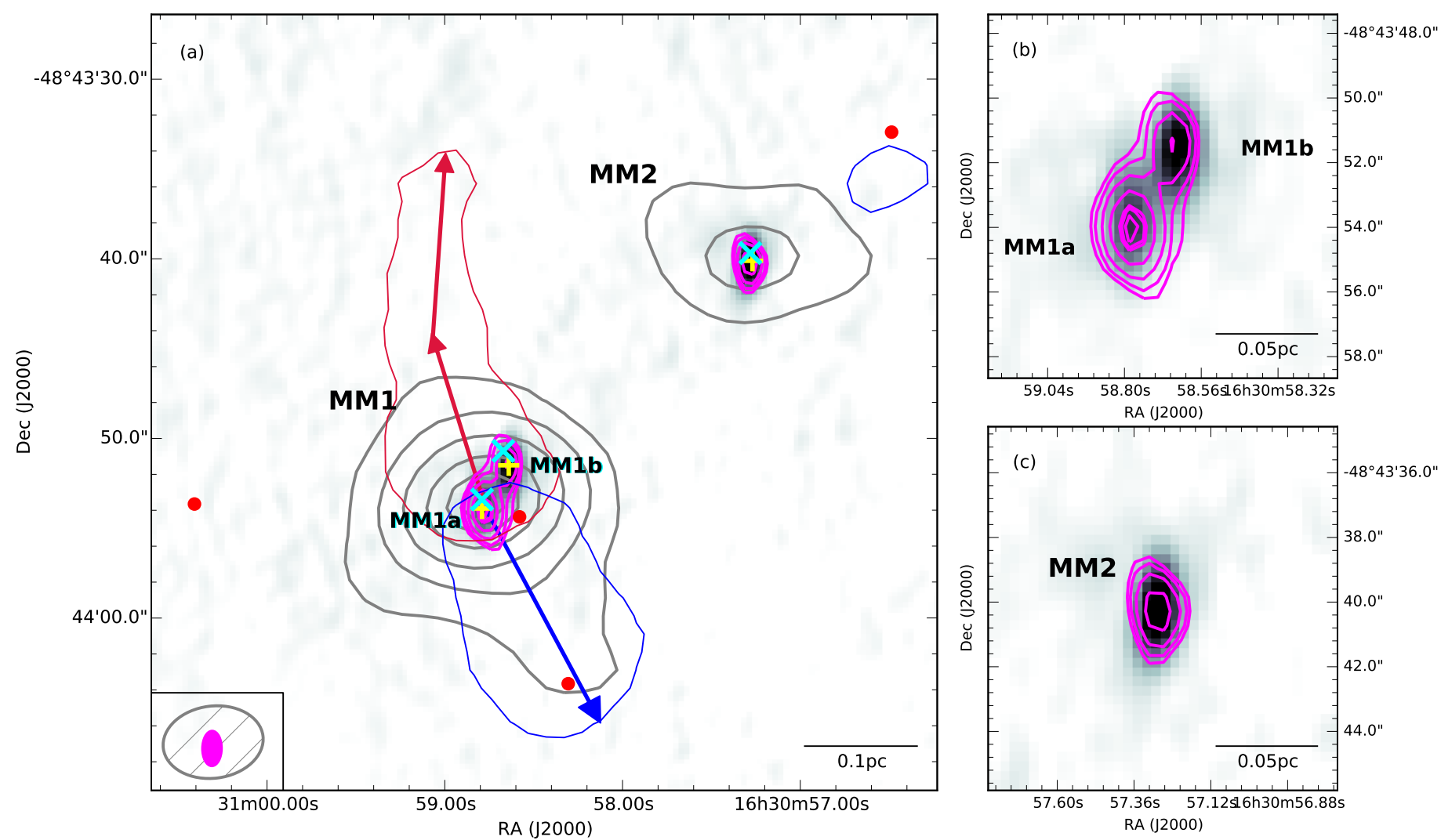

Fig. 3. a) SDC 335 IRDC as viewed in the radio/mm continuum. Colour-scale the $8 \mathrm{GHz}$ (ATCA) overlaid with ATCA $25 \mathrm{GHz}$ (magenta contours at 15,20,30, 50, 75, 80 and $90 \%$ of the peak flux) and the ALMA Cycle $03.2 \mathrm{~mm}$ continuum (grey contours at 2.5, 10, 25, 50, 75 and 90\% of

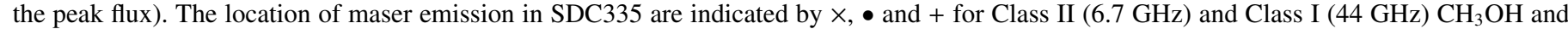
$\mathrm{H}_{2} \mathrm{O}(22 \mathrm{GHz})$ masers, respectively. The red and blue contours and red and blue arrows represent the 5\% of peak HNC emission boundary and direction of the HNC molecular outflow (Pineda et al., in prep., see main text for more detail). The synthesised beam shape for both the ALMA data (grey hatched) and ATCA $25 \mathrm{GHz}$ (solid magenta) are shown in the lower left. Panels b) and c): zoom in of the central regions of MM1 and MM2 respectively; both show 8 and $25 \mathrm{GHz}$ continuum as per main image.

Table 3. Positions of the radio cores observed in SDC335.

\begin{tabular}{ccc}
\hline \hline Source & \multicolumn{2}{c}{ J2000 } \\
& $\begin{array}{c}\text { Right ascension } \\
{[\mathrm{hh}: \mathrm{mm}: \mathrm{ss}]}\end{array}$ & $\begin{array}{c}\text { Declination } \\
{\left[{ }^{\circ} \mathbf{\prime}^{\prime} \mathrm{\prime}^{\prime \prime}\right]}\end{array}$ \\
\hline MM1 $a$ & $16: 30: 58.765$ & $-48: 43: 54.00$ \\
MM1 $b$ & $16: 30: 58.638$ & $-48: 43: 51.70$ \\
MM2 & $16: 30: 57.291$ & $-48: 43: 40.21$ \\
\hline
\end{tabular}

(Fish 2007), particularly the inner regions ( 6200 AU) thereof (Breen et al. 2010, and references therein) when the $\mathrm{H}_{2} \mathrm{O}$ and class II $\mathrm{CH}_{3} \mathrm{OH}$ masers are co-spatial. As such, each pairing of these maser species, one for each of the three radio continuum sources in SDC335, is indicative of the position of a forming massive star.

Class I $\mathrm{CH}_{3} \mathrm{OH}$ masers have been found to trace the larger scale molecular outflows of protostellar systems (Kurtz et al. 2004; Cyganowski et al. 2009). Two of the four class $\mathrm{I} \mathrm{CH}_{3} \mathrm{OH}$ maser spots in SDC335 inhabit the blue outflow arm originating from MM1a. A third spot is adjacent to the HNC blue lobe observed near MM2.

We consider these two sources independent objects given the assumed outflow origin, the angular offset between the outflow direction and a line connecting MM1 $a$ and MM1 $b$, and the presence of both an $\mathrm{H}_{2} \mathrm{O}$ and class $\mathrm{II} \mathrm{CH}_{3} \mathrm{OH}$ maser associated with the peak continuum flux of MM $1 a$ and $b$. Our analysis focusses on constraining the properties of MM1 1 , MM1 $b$ and MM2.
Table 4. Spectral index properties of the cores within SDC335.

\begin{tabular}{cccc}
\hline \hline$v$ & MM1 $a$ & MM1b & MM2 \\
interval & $\alpha$ & $\alpha$ & $\alpha$ \\
\hline $6-8 \mathrm{GHz}$ & $1.90 \pm 0.40$ & $1.01 \pm 0.12$ & $0.96 \pm 0.11$ \\
$8-23 \mathrm{GHz}$ & $1.67 \pm 0.20$ & $0.66 \pm 0.13$ & $0.67 \pm 0.10$ \\
$23-25 \mathrm{GHz}$ & - & - & $-0.11 \pm 0.02$ \\
\hline
\end{tabular}

Notes. We do not include the $\alpha$ value between 23 and $25 \mathrm{GHz}$ for sources MM1 $a$ and MM1 $b$, as it is possible the value is contaminated by some thermal emission (see Fig. 4a).

\subsection{Free-free component}

The three compact HII regions detected in our ATCA observations are observed with sufficient resolution to create partial SEDs from 6 to $25 \mathrm{GHz}$. These can be seen in Figs. $4 \mathrm{~b}$ and $\mathrm{c}$ and Fig. 5. We calculate for each source a spectral index, $\alpha$ (where $S_{\text {int }} \propto v^{\alpha}$ ), where $S_{\text {int }}$ is the source's integrated flux and $v$ frequency. The $\alpha$ values are listed in Table 4. One can consider $\alpha$ as an indicator of the free-free emission turnover frequency. The turnover frequency of free-free emission is the frequency at which a source transitions from optically thick to optically thin, i.e. where the source optical depth equals 1 . Below this frequency a free-free emission region is optically thick and $\alpha=2$, towards the turnover frequency $\alpha$ reduces and for optically thin sources $\alpha$ approaches -0.1 (Kurtz 2005). 

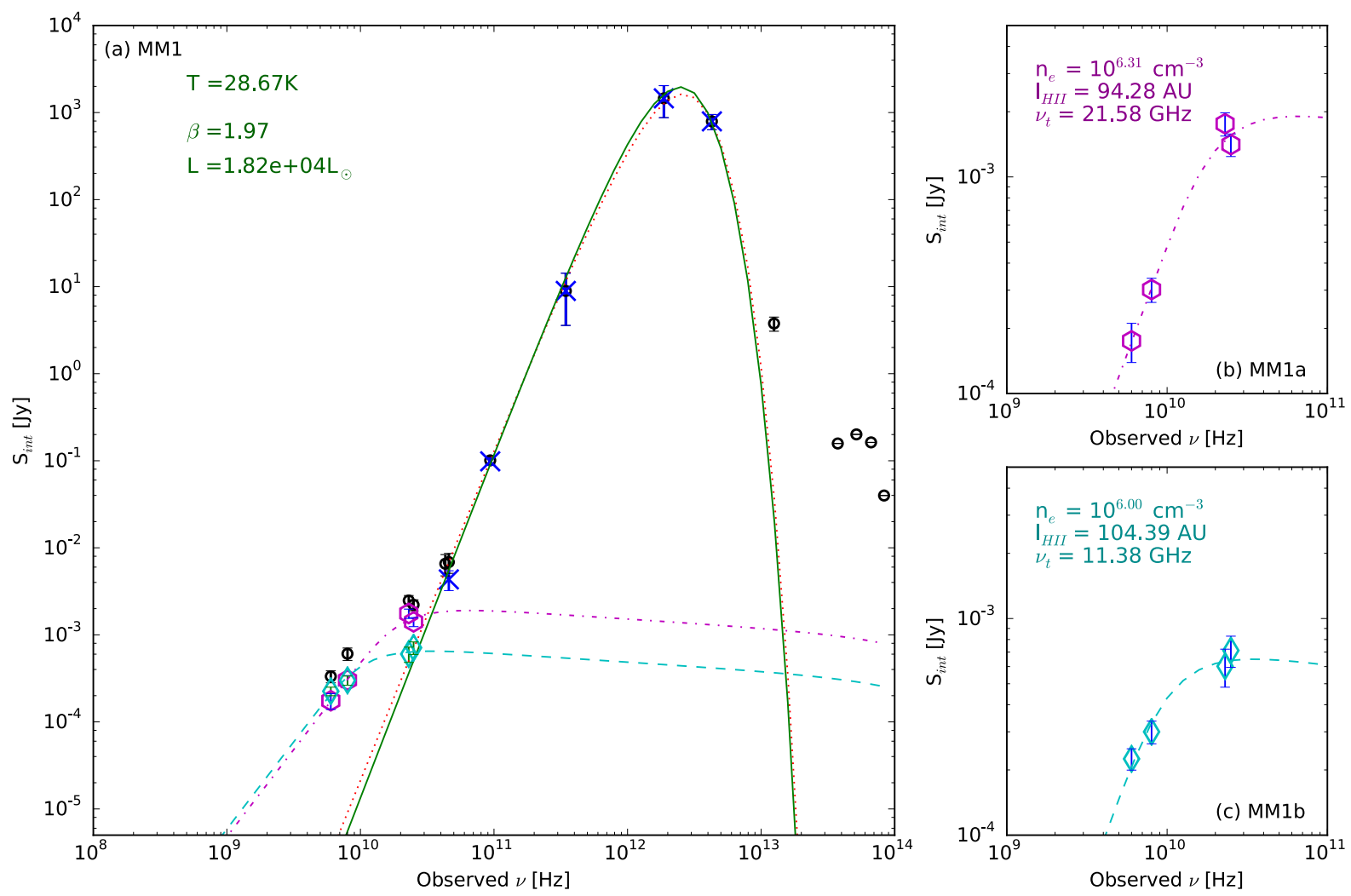

Fig. 4. Spectral energy distribution of the MM1 core on $\sim 5^{\prime \prime}$ scales. a) The black $\circ$ give the fluxes at each frequency, the blue $\times$ are the data used for the blackbody fit, note that at 23 and $25 \mathrm{GHz}$ the free-free component has be subtracted. The black-body fit with and without the free-free component subtraction are given by the solid green and red dotted lines respectively. The magenta dot-dash line and hexagons, and cyan dashed line and diamonds give the radio fluxes and follows the free-free fits for MM1 $a$ and MM1b, respectively. The free-free emission data for MM1 $a$ and MM1 $b$ can be seen separated in $\mathbf{b}$ ) and $\mathbf{c}$ ). (Colour online.)

Each source is optically thick between 6 and $8 \mathrm{GHz}$. MM1a appears to remain at least partially optically thick between 8 and $23 \mathrm{GHz}$ and the lower $\alpha$ values between these frequencies for MM1 $b$ and MM2 (compared to MM1 $a$ ) are also suggestive of partial optical thickness. However, these lower $\alpha$ values allow for the turnover frequency to occur between 8 and $23 \mathrm{GHz}$ for both MM1 $b$ and MM2.

MM2 is the only source for which there is insignificant contamination from thermal dust emission at $25 \mathrm{GHz}$, (contrast Figs. 4 and 5); the $\alpha$ value between 23 and $25 \mathrm{GHz}$ shows this HII region has become optically thin at these frequencies.

Based upon these spectral indices we consider the nature of the free-free emission, specifically considering the ambiguity between compact HII regions and collimated ionised jets at these resolutions. The steep spectral index of MM1 $a$ between 6-8 and 8-23 GHz is typical of that of a compact HII region (Kurtz 2005) and steeper than the expected value for collimated jet emission ( $\alpha=0.25-1.1$, Reynolds 1986). Thus we classify MM1 $a$ as a hyper-compact HII (HCHII) region.

The spectral indices of MM1 $b$ and MM2 of $\sim 1.0$ and $\sim 0.6$ at 6-8 and 8-23 GHz in both sources, respectively, fall within the characteristic range of both collimated jet emission (Reynolds 1986) and photo-ionised compact HII regions in the transition from optically thick to thin. In the case of MM1 $b$, given its misalignment to the molecular outflow originating from MM1 $a$ and centrally located (potentially disc tracing) $\mathrm{CH}_{3} \mathrm{OH}$ maser, we assume MM1 $b$ is not part of a radio jet within the MM1 mmcore and instead favour the interpretation that this source is also a compact HII region ionised by a separate source to MM1a. We also assume the compact HII region interpretation for MM2

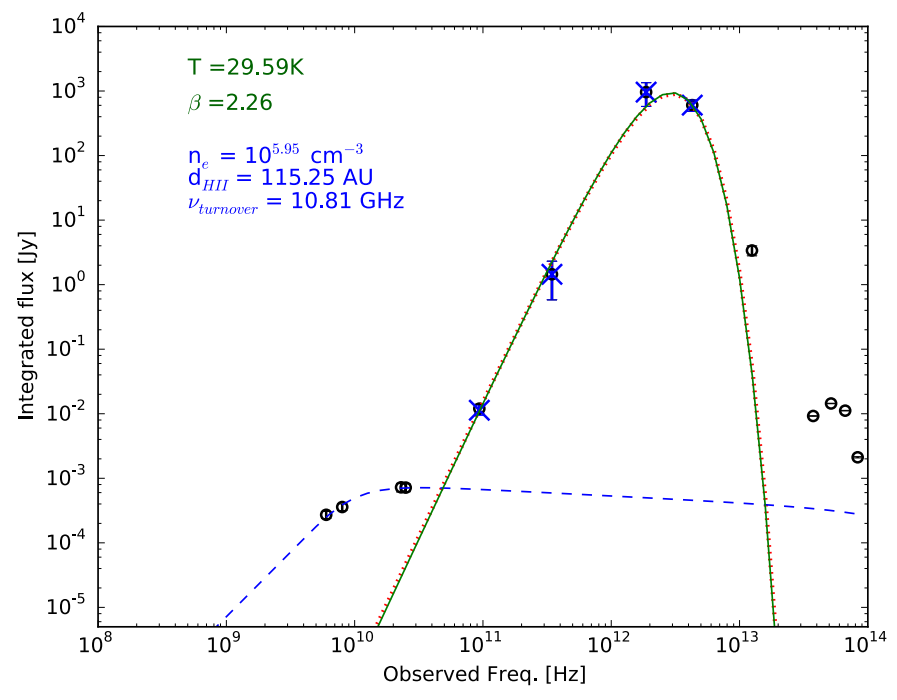

Fig. 5. Spectral energy distribution of the MM2 core. Symbols as Fig. 4a. (Colour online.)

based on the lack of a firm detection of a nearby strong collimated outflow. However, within the scope of these data either the radio jet or compact HII region interpretation may be valid for both MM1 $b$ and MM2. The ultimate result of this ambiguity is that the stellar mass limits calculated assuming a compact HII region, ergo a photoionisation origin for the radio emission, will not hold true for radio jets. However the association of MM1 $b$ 
Table 5. Properties of the cores within SDC335 from the SED fitting.

\begin{tabular}{|c|c|c|c|c|c|c|c|c|}
\hline Object & $\begin{array}{c}E M \\
{\left[\mathrm{pc} \mathrm{cm}^{-6}\right]}\end{array}$ & $\begin{array}{c}n_{\mathrm{e}} \\
{\left[\mathrm{cm}^{-3}\right]}\end{array}$ & $\begin{array}{c}l_{H \mathrm{II}} \\
{[\mathrm{AU}]} \\
\end{array}$ & $\begin{array}{c}N_{\text {Lym }}^{\dagger} \\
\text { [photon s }^{-1} \text { ] }\end{array}$ & $\begin{array}{c}L_{\text {bol }} \\
{\left[\mathrm{L}_{\odot}\right]}\end{array}$ & $\begin{array}{c}T_{\mathrm{d}} \\
{[\mathrm{K}]}\end{array}$ & $\beta$ & $\begin{array}{l}M_{\mathrm{gas}} \\
{\left[M_{\odot}\right]} \\
\end{array}$ \\
\hline MM1 & - & - & - & - & $1.8 \times 10^{4}$ & $28.7( \pm 2.3)$ & $1.97( \pm 0.15)$ & $763\left({ }_{-171}^{+165}\right)$ \\
\hline$M M 1_{\mathrm{p}}$ & - & - & - & - & $1.6 \times 10^{4}$ & $30.0( \pm 1.7)$ & $1.75( \pm 0.11)$ & $429\left({ }_{-109}^{+104}\right)$ \\
\hline MM1a & $1.9( \pm 0.5) \times 10^{9}$ & $2.1( \pm 0.3) \times 10^{6}$ & $94.28( \pm 5.7)$ & $>2.0\left({ }_{-0.5}^{+0.5}\right) \times 10^{45}$ & - & - & - & - \\
\hline$M M 1 a_{\mathrm{p}}$ & $2.0( \pm 0.4) \times 10^{9}$ & $2.1( \pm 0.2) \times 10^{6}$ & $93.96( \pm 7.1)$ & $"$ & - & - & - & - \\
\hline MM1b & $5.0( \pm 1.0) \times 10^{8}$ & $1.0( \pm 0.1) \times 10^{6}$ & $104.4( \pm 5.2)$ & $6.8\left({ }_{-2.0}^{+1.9}\right) \times 10^{44}$ & - & - & - & - \\
\hline MM2 & $4.5( \pm 0.5) \times 10^{8}$ & $8.9( \pm 0.5) \times 10^{5}$ & $115.2( \pm 4.7)$ & $8.2\left({ }_{-2.0}^{+1.9}\right) \times 10^{44}$ & $9.9 \times 10^{3}$ & $29.6( \pm 7.2)$ & $2.26( \pm 0.52)$ & $173\left({ }_{-66}^{+65}\right)$ \\
\hline
\end{tabular}

Notes. ${ }^{(\dagger)} N_{\text {Lym }}$ values are lower limits, see text. The values shown in rows $M M 1_{\mathrm{p}}$ and $M M 1 a_{\mathrm{p}}$ are those which include the $43 \mathrm{GHz}$ data point in the free-free emission SED fitting, see Sect. 3.2.2 for more details.

and MM2 with class II $\mathrm{CH}_{3} \mathrm{OH}$ masers confirms that they are forming massive stars.

\subsubsection{Free-free fitting technique}

To further this analysis we fit the free-free component using the 6, 8, 23 and $25 \mathrm{GHz}$ ATCA data, implementing a non-linear least squares regression algorithm. The fitted parameters are the freefree turnover frequency, $v_{\mathrm{t}}$, (where the optical depth, $\tau=1$ ) and the physical diameter of the source, $l$. Setting $\tau=1$ and fixing $T_{\mathrm{e}}$ to the fiducial $10000 \mathrm{~K}$ (e.g. Spitzer 1978; Sánchez-Monge et al. 2011), emission measure ( $E M)$, we calculated values for a given turnover frequency using the approximate formula for the optical depth of free-free emission (Mezger \& Henderson 1967, and references therein):

$\tau_{\mathrm{ff}} \approx 0.08235 T_{\mathrm{e}}^{-1.35}\left(\frac{v}{\mathrm{GHz}}\right)^{-2.1}\left(\frac{E M}{\mathrm{pc} \mathrm{cm}^{-6}}\right)$.

Based on this $E M$ value the optical depth, $\tau_{\mathrm{ff}}$, following Eq. (1), was used in Eq. (2),

$S_{\mathrm{ff}-\mathrm{fit}}=\Omega_{\mathrm{ff}} B_{v}\left(T_{\mathrm{e}}\right)\left(1-\mathrm{e}^{-\tau_{\mathrm{ff}}}\right)$,

to fit the flux density of the data. Here $\Omega_{\mathrm{ff}}$ is the solid angle subtended by the source and $B_{v}\left(T_{\mathrm{e}}\right)$ the Planck distribution value at a given frequency at $T_{\mathrm{e}} . S_{\mathrm{ff}-\mathrm{fit}}$ was then fit to the observed fluxes with the best fit giving values for $v_{\mathrm{t}}, E M$ and $l$. The $E M$ value calculated at the turnover frequency is independent of any knowledge of the source's physical dimensions meaning there is no degeneracy in the value of $l$, which is only used in calculating the value of $\Omega_{\mathrm{ff}}$.

\subsubsection{Fitted properties}

The fitted properites for the three HII regions seen in SDC335 are given in Table 5. Each object has fitted size of $<0.5 \mathrm{mpc}$ $(\simeq 110 \mathrm{AU})$, a value much smaller than that of the upper size limit associated with the hyper-compact HII region classification ( $\$ 30$ mpc e.g. Kurtz 2005). Sources of similar size have been observed in high mass star-forming regions, e.g. W3 IRS 5 (Wilson et al. 2003) with sources $\mathrm{d} 1 / \mathrm{d} 2$ and $\mathrm{f}$ both measuring deconvolved radii of $0.8 \mathrm{mpc}(\simeq 165 \mathrm{AU})$ using the VLA at 1.3 and $0.7 \mathrm{~cm}$. Zhu et al. (2013) observed the HCHII region NGC 7538 IRS1 with the SMA and CARMA (at 1.3 and $3.4 \mathrm{~mm}$ ) and resolved it into a compact component $<270 \mathrm{AU}(\simeq 1.3 \mathrm{mpc})$ and an extended tail of $2000 \mathrm{AU}(\simeq 9 \mathrm{mpc})$. Also, Sánchez-Monge et al. (2011) in their VLA search for new HCHII regions (in a methanol maser selected sample) found six new HCHII candidates with derived sizes of between 1 and $10 \mathrm{mpc}(\simeq 206-$ 2060 AU).

The fitted optically thick to optically thin turn over frequencies for the three SDC335 HII regions are in agreement with the values expected from the spectral indices calculated between each of our observing frequencies. MM1 $a$ is the densest of the 3 HII regions in SDC335, remaining optically thick to frequencies $\geq 20 \mathrm{GHz}$. MM1 $b$ and MM2 exhibit similar properties to one another with fitted turnovers of $\sim 11 \mathrm{GHz}$. From the fitted values for each of our compact radio sources it seems likely that SDC335 harbours three HCHII regions. The fit to MM1a is the least robust as the data for this source allows for a rise in flux beyond $25 \mathrm{GHz}$. However, the fitting technique limits the turnover frequency to be within the frequency range of the data. This issue is addressed further in Sect. 3.2.2.

\subsubsection{Derived properties}

For each source we calculated an electron number density, $n_{\mathrm{e}}$, and the number density of Lyman $\alpha$ photons being emitted. The Lyman $\alpha$ photon number density allows the association of the three HCHII regions with a zero-age main sequence (ZAMS) stellar spectral type. Assuming a cylindrical homogeneous HII region $E M$ is related to electron number density by $E M=\int_{0}^{l} n_{\mathrm{e}}^{2} \mathrm{~d} l \approx n_{\mathrm{e}}^{2} l$. Calculated values for $n_{\mathrm{e}}$ are found in Table 5 and again exhibit values associated with HCHII $\left(\gtrsim 10^{6} \mathrm{~cm}^{-3}\right)$.

The Lyman continuum flux assuming optically thin emission is given by

$$
\begin{aligned}
N_{\text {Lym }}= & 7.6 \times 10^{46} \alpha^{-1}\left(v, T_{\mathrm{e}}\right)\left(\frac{T_{\mathrm{e}}}{10^{4} \mathrm{~K}}\right)^{0.35} \\
& \times\left(\frac{v}{\mathrm{GHz}}\right)^{0.1}\left(\frac{S_{\text {int,cont }}}{\mathrm{Jy}}\right)\left(\frac{D}{\mathrm{kpc}}\right)^{2.0} \text { photons s }^{-1}
\end{aligned}
$$

(Zhu et al. 2013), where $S_{\text {int,cont }}$ is the radio continuum flux density at a given frequency and $D$ the distance to SDC335 (i.e. $3.25 \mathrm{kpc}$ ). For each source we calculate $N_{\text {Lym }}$ using the $23 \mathrm{GHz}$ continuum integrated flux density value. It is important to note that as MM1a is optically thick beyond $25 \mathrm{GHz}$, use of the 23 (or indeed 25) $\mathrm{GHz}$ will lead to an underestimate in $N_{\text {Lym }}$ for this source, meaning that our calculated value is a lower limit. As we indicated previously, we set $T_{\mathrm{e}}=10000 \mathrm{~K}$, giving $\alpha\left(v, T_{\mathrm{e}}\right)=0.966$ (Mezger \& Henderson 1967). 
The $N_{\text {Lym }}$ values were then compared with the Lyman continuum values for ZAMS stars given in Davies et al. (2011) and spectral types from Mottram et al. (2011). From these values, the protostellar cores in SDC335 appear to have currently formed a single B1.5 $\left(M_{*}=9.0 M_{\odot}\right)$ type star in the MM2 core, while the MM1 core has formed at least two stars one of B1.5 (MM1b) spectral type and a second of at least spectral type B1-B1.5 $\left(M_{*}=10.0 M_{\odot}\right)$ for MM1 $a$. These spectral classifications are based on the properties of the protostars as they are observed now if they were at the ZAMS stage and not still actively accreting.

\subsection{Spectral energy distribution of SDC335 protostellar cores}

Combining the multi-frequency data available (from this work and ancillary data described in Sect. 2.2), and listed in Table 2, we create spectral energy distributions (SEDs) for the dust emission from MM1 and MM2. These were created by first subtracting the best-fit free-free emission from the thermal emission at all frequencies used in the fit $(25 \mathrm{GHz}$ to $4.3 \mathrm{THz}(70 \mu \mathrm{m})$ for each source) and then fitting the SED to these corrected data.

For the purpose of SED fitting, MM1 is treated as two objects (MM1 $a$ and MM1 $b$ ) at frequencies between 6 and $25 \mathrm{GHz}$, and as a single object above these frequencies where the resolution is $5^{\prime \prime}$ or greater. As the respective resolutions of the data used in this fitting vary as a function of frequency, we conservatively compensate for potential inclusion of excess flux in lower resolution data. To achieve this, the uncertainties of the data points (presented in Table 2) with beams greater than $6^{\prime \prime}$ have been scaled assuming a density profile of $r^{-2}$ for these sources (see Peretto et al. 2013, Table 1).

The modified blackbody fitted is of the form:

$S_{\mathrm{bb}-\mathrm{fit}}=\Omega_{\mathrm{c}} B_{v}\left(T_{\mathrm{d}}\right)\left(1-\mathrm{e}^{-\tau_{\mathrm{bb}}}\right)$

where $B_{v}\left(T_{\mathrm{d}}\right)$ is the Planck distribution value at a given frequency, $v$, and a dust temperature $T_{\mathrm{d}} . \tau_{\mathrm{bb}}$ is the optical depth of dust and has the form $\left(\frac{v}{v_{0}}\right)^{\beta}$, with $v_{0}$ as the frequency at which the optical depth is unity. Finally, $\Omega_{c}$ is the solid angle subtended by the dust-dominated region. For the purposes of fitting we fix $\Omega_{\mathrm{c}}$ to the area subtended from the Herschel data using the Hyper photometry code (Traficante et al. 2015) which has radius $11.4^{\prime \prime}$.

The free parameters in our fitting routine are, $T_{\mathrm{d}}, v_{0}$, and $\beta$, and are given priors of $25.0 \mathrm{~K}, 4.3 \mathrm{THz}$, and 2.0 respectively. The resulting best fit parameters are then used in a direct integration of the SED between $100 \mathrm{kHz}$ and $100 \mathrm{THz}$ ( $3 \mathrm{~km}$ to $3 \mu \mathrm{m})$ to calculate the source bolometric luminosity. We also refine the mass of the mm-cores MM1 and MM2 using the $3.2 \mathrm{~mm}$ ALMA flux densities (see Table 2), at deconvolved size scales of $0.054 \mathrm{pc}$ and $0.057 \mathrm{pc}$ (following Peretto et al. 2013), applying our fitted $T_{\mathrm{d}}, v_{0}$, and $\beta$ in the mass flux relation

$M_{\text {gas }}=\frac{D^{2} S_{3.2}}{\kappa_{3.2} B_{3.2}\left(T_{\mathrm{d}}\right)}$

using the dust opacity law $\kappa_{\lambda}=0.1 \times\left(\frac{\lambda}{0.3 \mathrm{~mm}}\right)^{-\beta} \mathrm{cm}^{2} \mathrm{~g}^{-1}$, assuming a dust to gas ratio of 0.01 (Hildebrand 1983; Beckwith et al. 1990).

\subsubsection{SED fitting results}

Table 5 presents the parameters derived from our SED fitting. Overall the fitted and derived values from our SEDs are typical of those of a massive protostellar core in early stages of star formation (e.g. Rathborne et al. 2008; Beuther et al. 2010; Rathborne et al. 2010).

\subsubsection{Posteriori fitting of MM1a}

As noted in Sect. 3.1.1, the free-free emission from MM1 $a$ is the least well constrained with the turnover potentially occurring above the $25 \mathrm{GHz}$ data point used in the initial fit. To attempt to overcome this we use the SED fitted results for MM1 ( $T=28.7 \mathrm{~K}$ and $\beta=1.97$ ) following the above method. We then adjust the $43 \mathrm{GHz}$ data point and refit the free-free emission from MM1 $a$ including this data point. The model fit of MM1 $b$ is kept as constant within these fits. The fitting of free-free emission data and then the full (free-free subtracted) SED proceeds iteratively until best-fit values for MM1 $a$ in free-free $\left(l, v_{\mathrm{t}}\right.$, and $E M)$ and new best-fit values for MM1 as a single object ( $T$ and $\beta$ ) are found. These new values can be found in Table 5 labelled subscript " $p$ " and represented in italics.

The new fitted properties for MM1 and MM1 $a$ are consistent with the initial fitted values for each source, which may suggest a robust fit to these data or highlight the detrimental effect of the larger error associated with the $43 \mathrm{GHz}$ data point compared to those from the primary data (6-25 GHz ATCA) for this work. As such we consider only the initial fit values throughout the remainder of this work.

\section{Discussion}

\subsection{The evolutionary status of the ionising sources in SDC335}

In Sect. 3.1.3 we calculate a Lyman photon flux for each HCHII and from this associate a ZAMS spectral type to the source ionising each HCHII. We now consider the evolutionary status of the three ionising sources in SDC335 with respect to the calculated $L_{\mathrm{bol}}$ values for the MM1 and MM2 cores (Sect. 3.2.1) and the protostellar evolutionary tracers associated with each source compared to the characteristics that the ZAMS spectral type would apply.

A star arrives at the ZAMS once hydrogen burning has commenced. Within SDC335, we expect accretion to be ongoing, as illustrated by the presence molecular outflow from the MM1 mm-core (Pineda et al., in prep.), and each ionising source exhibiting a $22 \mathrm{GHz}$ water maser (Breen et al. 2010; Avison et al., in prep.), a maser species associated with outflows from both low and high mass young stellar objects (Fish 2007). In addition to these, an extended green object (EGO; Cyganowski et al. 2008), another outflow tracer, is also present at the centre of SDC335 filamentary structure. The global collapse of SDC335 also supports the ongoing accretion of the protostars ionising the three detected HCHII.

Accretion rates of between $\geq \times 10^{-4}$ and up to a few $\times 10^{-3} M_{\odot} \mathrm{yr}^{-1}$ (e.g. Fuller et al. 2005; Churchwell et al. 2010; Klaassen et al. 2012; Rygl et al. 2013; Duarte-Cabral et al. 2013) have been calculated for massive star precursors (e.g. HMPOs, HCHII), a greater value than that associated with their lower mass counterparts $\left(\dot{M}_{*} \sim \frac{c_{\mathrm{s}}^{3}}{G} \simeq 5 \times 10^{-6} M_{\odot} \mathrm{yr}^{-1}\right.$; Shu 1977; McKee \& Tan 2003). Given these observed values, we make the assumption that the infall rate of SDC335 $(2.5 \pm$ 1.0) $\times 10^{-3} M_{\odot} \mathrm{yr}^{-1}$ (Peretto et al. 2013) continues unimpeded onto the protostars. During the accretion phase of a young stellar object the total output luminosity is comprised of an accretion component and the intrinsic luminosity, $L_{\mathrm{tot}}=L_{*}+L_{\mathrm{acc}}$ with 
Table 6. Calculated luminosity values for MM1 $a$, MM1 $b$, and MM2 using the assumed ZAMS properties of each ionising source.

\begin{tabular}{cccc}
\hline \hline Source & $L_{*}$ & $\begin{array}{c}L_{\text {acc }} \\
{\left[L_{\odot}\right]}\end{array}$ & $\begin{array}{c}L_{\text {tot,ZAMS }} \\
{\left[L_{\odot}\right]}\end{array}$ \\
\hline MM1 $a$ & $5.48 \times 10^{3}$ & $2.22 \times 10^{5}$ & $2.27 \times 10^{5}$ \\
MM1 $b$ & $4.10 \times 10^{3}$ & $2.16 \times 10^{5}$ & $2.20 \times 10^{5}$ \\
MM2 & $4.35 \times 10^{3}$ & $2.17 \times 10^{5}$ & $2.21 \times 10^{5}$ \\
\hline
\end{tabular}

the accretion component taking the form $L_{\mathrm{acc}}=\frac{G M_{*} \dot{M}_{*}}{R_{*}}$. If we assume the protostars have the ZAMS radii, $R_{*}$ and luminosity, $L_{*}$ interpolated from Davies et al. (2011, Table 1) to our calculated $N_{\text {Lym }}$ values and use the ZAMS mass-luminosity relation of $L_{*} \propto M_{*}^{3.5}$, we can calculate a mass for each protostar. With these values, we then calculate $L_{\text {tot }}$ for MM1 and MM2 (in the case of MM1 the value is the addition of calculated values for MM1 $a$ and MM1 $b$ ). In both cases $L_{\mathrm{tot}}$ exceeds the calculated $L_{\mathrm{bol}}$ by a factor $\sim 20$, with $L_{\text {acc }}$ the dominant luminosity (see Table 6 ).

Initially, we note that the ZAMS $L_{*}$, without the additional luminosity contribution from $L_{\mathrm{acc}}$, for both MM1 and MM2 are within a factor $\sim 2$ of the measured $L_{\mathrm{bol}}$ for both MM1 and MM2, which is not a large discrepancy given the uncertainties in the data. However, the number of outflow indicators associated with the protostars (as noted above) excludes the scenario in which the SDC335 protostars have ceased accretion. Instead we consider two mechanisms by which the discrepancy between $L_{\text {tot,ZAMs }}$ and $L_{\text {bol }}$ can be addressed.

First, we consider the models for massive protostellar evolution with high accretion rates $\left(\geq 10^{-3} M_{\odot} \mathrm{yr}^{-1}\right)$ presented by Hosokawa \& Omukai (2009) and Hosokawa et al. (2010) for spherical collapse and accretion via a disc, respectively. Under both scenarios, massive protostars transition through a number of stages before joining the ZAMS. These stages include swelling at masses $6 \lesssim M_{*} \lesssim 10 M_{\odot}$ and contracting again between $10 \lesssim M_{*} \lesssim 30 M_{\odot}$, (for a constant $\dot{M} \sim 10^{-3} M_{\odot} \mathrm{yr}^{-1}$ ). During the swelling phase the protostellar radii swells rapidly from a few $R_{\odot}$ to $\sim 100 R_{\odot}$ whilst the intrinsic luminosity also increases. As a result of the swelling the effective temperature of the protostar is lower than that of a ZAMS star with the same luminosity, with temperatures low enough that there are insufficient photons with energies greater than $13.6 \mathrm{eV}$ to create a HII region.

Again following the $\dot{M} \sim 10^{-3} M_{\odot} \mathrm{yr}^{-1}$ case reported on for both accretion scenarios in Hosokawa \& Omukai (2009) and Hosokawa et al. (2010), after the swelling of the protostar, a period of contraction commences whilst accretion continues. The $L_{*}$ becomes the dominant component of $L_{\text {tot }}$ over $L_{\text {acc }}$ and $R_{*}$ drops from $\sim 100 R_{\odot}$ to of the order $10 R_{\odot}$ as the protostar increases in mass. It is during this phase that the protostellar effective temperature becomes high enough that the flux of ionising photons is sufficient to start the formation of a HII region.

Under this high accretion rate scenario and given that our protostars are displaying ionisation but have $L_{\mathrm{bol}}<L_{\mathrm{tot}, \mathrm{ZAMS}}$, we suggest the possibility that the protostars within SDC335 are in the contraction phase of their formation, putting their current masses each at $\geq 10 M_{\odot}$ (see e.g. Hosokawa \& Omukai 2009, Fig. 18) with their eventual final ZAMS mass higher than this. It is interesting to note that our assumed mass accretion rate $(2.5 \pm 1.0) \times 10^{-3} M_{\odot} \mathrm{yr}^{-1}$ approaches the limiting case of the Hosokawa et al. (2010) model at $\sim 4 \times 10^{-3} M_{\odot} \mathrm{yr}^{-1}$, an accretion rate value at which a second stage of rapid radial expansion occurs halting contraction and steady accretion ceases. In this case the temperature again decreases owing to the radial expansion and no HII regions are created. This provides a maximum limit on the accertion rates of these sources of $\lesssim 4 \times 10^{-3} M_{\odot} \mathrm{yr}^{-1}$ (Hosokawa et al. 2010).

The second mechanism that can address the luminosity discrepancy is that there is a decreased, decreasing or periodic accretion when compared to the $\dot{M}_{*}$ used in our $L_{\text {acc }}$ calculation. The value $\dot{M}_{*}=2.5 \times 10^{-3} M_{\odot} \mathrm{yr}^{-3}$ used was found by Peretto et al. (2013) and assumes spherical accretion onto the central mm-cores of SDC335 due to the global cloud collapse. It is possible that the accretion rate onto the protostars is lower than onto the cores, meaning that the cores are growing at a greater rate than the protostars, and thus $L_{\text {acc }}$ is lower. Indeed, reducing the accretion rate onto the protostars by the factor 20 gives an $\dot{M}_{*}$ of $1.25 \times 10^{-4} M_{\odot} \mathrm{yr}^{-3}$, which is not unreasonable for a forming massive star.

Decreasing or periodic accretion onto the protostars would lead to fluctuations in $L_{\text {acc }}$ over time. These are the most difficult scenarios to address in any quantitative manner as it requires both knowledge of the accretion onto the sources in a more direct way than our current data allow and preferably observation of this accretion or suitable proxies at more than one epoch. Opposing these accretion rate scenarios Davies et al. (2011) found in their population synthesis based on the Red MSX Source survey (RMS) data (Hoare et al. 2004) that declining (or indeed constant) accretion rates as a function of time do not well fit the observed massive star population of the galaxy. There is currently no concensus on periodic accretion onto massive protostars within the literature.

Our current data on SDC335 do not allow us to select the likely candidate from these scenarios as being the mechanism responsible for the lower than expected $L_{\mathrm{bol}}$. Further observation of the SDC335 protostars targeted at constraining the accretion on smaller scales is required to resolve this issue.

\subsection{Cluster formation in SDC335}

Given that we have discovered three massive protostars within SDC335 it is possible to put limits on the eventual number of stars the IRDC is capable of creating. We employ the power-law form of the IMF, $N \propto M^{-\alpha}$, with $\alpha=2.35$ (Salpeter 1955) and $\alpha=2.3$ (Kroupa 2002) in the standard mass range of 1-120 $M_{\odot}$. With the criteria that three objects have masses above $9.7 M_{\odot}$, we find that SDC335 has the potential to form a minimum of 54-60 stars (between 1 and $120 M_{\odot}$ ).

Continuing the Kroupa (2002) IMF down to a mass of $0.08 M_{\odot}$ SDC335 has the potential to form $\sim 1400$ stars, with the vast majority of these $(\sim 96 \%)$ in the sub-solar mass regime. Using the average stellar mass from Kroupa (2002) Table 2, this would give an approximate total mass in stars, $M_{\star \text { tot }}$, of $\sim 285 M_{\odot}$, a small fraction (5\%) of the total mass of the SDC335 cloud $\left(5500 \pm 800 M_{\odot}\right)$. Assuming a typical star formation efficiency of $\sim 10-30 \%$ for embedded clusters (Lada \& Lada 2003) one would expect a $M_{\star \text { tot }}$ from SDC335 in the range 550$1650 M_{\odot}$. Keeping in mind the uncertainties on our mass estimates, the discrepancy between this and our IMF calculated value could be the result of our underestimation of the true final masses of the three massive protostellar objects in SDC335.

\subsubsection{The future of SDC335}

For a young system such as SDC335 in the early stages of its star formation process it is interesting to make comparisons to systems with similar characteristics at different stages of evolution. 
One such system is the Trapezium cluster (TC) at the centre of the Orion nebula cluster (ONC). This extensively studied relatively evolved system (see e.g. Hillenbrand 1997; Muench et al. 2002) has some obvious similarities to our younger system. Centered on $\theta^{1} C$ Ori, TC has a diameter of $\sim 0.3 \mathrm{pc}$ (Hillenbrand 1997) comparable to the separation of the two SDC335 millimetre cores $(0.32 \mathrm{pc})$. The eponymous Trapezium stars comprise five $\mathrm{OB}$ stars at the heart of the cluster similar to the three protostars exciting the HCHII regions described in this work. Extending out to include the inner $3 \mathrm{pc}$ (and encompassing the $\mathrm{TC}$ ) is the ONC, a region of physical size comparable to the whole of SDC335 ( 2.4 pc).

Beyond these obvious similarities, the stellar population study of the ONC as reported by Hillenbrand (1997) detects $\sim 1600$ optically visible stars, with an expected additional $50 \%$ not detected because of their embedded nature. Of these sources, the authors report on the $60 \%$ of stars for which photometry and spectral types are available, which allows these objects to be associated with a mass. In the mass range 1-50 $M_{\odot}$ their sample is complete and contains 137 objects with 6 stars of mass greater than $10 M_{\odot}$, (the Trapezium stars plus others at a larger distance from $\theta^{1} C$ Ori). We have shown SDC335 has 3 stars of mass $\gtrsim 10 M_{\odot}$ and we expect 60 stars in a similar mass range to the 137 stars in ONC. From this it would appear we can expect a similar population in SDC335 to that in the ONC to within a factor $\sim 2$.

In SDC335 all three HCHII regions are found in the IRDCs central region (at the confluence of the filamentary arms). Hillenbrand (1997) finds evidence for mass segregation in the more evolved ONC with the most massive stars preferentially located within projected radii of $<0.3$ pc. In a later paper Hillenbrand \& Hartmann (1998) find that this distribution of stars is unlikely to be the result of dynamical mass segregation since its timescale is larger than the age of the ONC stars. The authors instead favour a primordial origin of the observed mass segregation. This interpretation is consistent with our result that in, SDC335, the three most massive sources lie at the bottom of the gas-dominated, gravitational potential well.

A second comparable region is the NGC $6334 \mathrm{I}(\mathrm{N})$ protocluster, which has been well studied at $\mathrm{mm}$ wavelengths, most recently by Hunter et al. (2014). NGC $6334 \mathrm{I}(\mathrm{N})$, hereafter simply $\mathrm{I}(\mathrm{N})$, is an IRDC at a distance of $1.3 \mathrm{kpc}$ and displays seven compact millimetre continuum sources within a projected diameter of $0.1 \mathrm{pc}$ at $2^{\prime \prime}$ resolution. In addition, in their study Hunter et al. (2014) find 25 likely protostellar objects associated within a physical radius of $0.21 \mathrm{pc}$ at $1.3 \mathrm{~mm}$.

There are four radio $(5 \mathrm{GHz})$ continuum sources within the same $0.21 \mathrm{pc}$ radius area, three of which are labelled as protostellar candidates and two are directly associated with $1.3 \mathrm{~mm}$ cores. The limited number of radio continuum detections in $\mathrm{I}(\mathrm{N})$ has an interesting corollary with SDC335, scaling the fluxes of the $\mathrm{I}(\mathrm{N})$ radio continuum detections to the distance of SDC335 we find that two of the four $\mathrm{I}(\mathrm{N})$ sources would be observed as $5 \sigma$ or greater in SDC335. Given this and the fact we observe three radio continuum sources in SDC335, if we assume I(N) and SDC335 have similar protostellar population density profiles one would expect a similar number of compact mm sources to exist in SDC335. However, currently the comparatively low resolution of the Peretto et al. (2013) ALMA data at $3.2 \mathrm{~mm}$ compared to that of the $1.3 \mathrm{~mm}$ observations of $\mathrm{I}(\mathrm{N})$, coupled with the fact that these two mm frequencies will trace different regions around the protostellar populations therein, means we are limited to making a qualitative comparison, given our current knowledge of the mm-core population of SDC335.
Qualitative comparisons of SDC335 to the NGC 6634 I(N) protocluster and the relatively evolved Trapezium cluster indicates that SDC335 is likely to form a similar population of stars. Further investigation of SDC335 with higher resolution at mm wavelength observations will allow the lower mass/non ionising core population to be detected making these comparisons more robust.

\section{Conclusions}

We have presented the results of radio continuum observations conducted with the ATCA towards the infrared dark cloud SDC335. Within the two massive protostellar cores observed by ALMA in SDC335 we have observed three compact HII regions. Fitting of the free-free emission of these sources indicates that they have characteristics typical of hyper-compact HII regions. Based on the fitted and derived characteristics of these HCHII regions, we find that the three protostars exciting them display characteristics of ZAMS stars of at minimum, spectral type B1.5 for two of the three, and B1-1.5 for the third. This can be considered a lower limit of their characteristics as accretion is still ongoing within SDC335.

With the use of ancillary data, we created free-free component subtracted SEDs that enable the calculation of the bolometric luminosities for the MM1 (treated as a single object at frequencies above $25 \mathrm{GHz}$ ) and MM2 cores. We find values from our SED fits to each HCHII region and mm-core that are typical of massive protostellar sources.

Our observed luminosities for the two cores are lower than would be expected from the ZAMS classification derived from the ionised flux. We interpret this result as a consequence of one of two mechanisms reducing the contribution of accretion to the total luminosity. First, the protostars may be at an early stage of accretion, still with an enlarged but contracting stellar radii after the "swollen core" stage for high accretion rate objects $\left(\dot{M}_{*} \geq\right.$ $10^{-4} M_{\odot} \mathrm{yr}^{-1}$; Hosokawa \& Omukai 2009), indicating that the protostellar objects exciting our HCHII regions will ultimately become more massive than their current ionising flux indicates. Secondly, the assumed accretion rate onto the protostars within the mm-cores MM1 and MM2 may be lower than onto the cores themselves.

We have drawn comparisons between SDC335 and both the relatively evolved Trapezium cluster and the evolving NGC 6334 $\mathrm{I}(\mathrm{N})$ protocluster to highlight the similarities of SDC335 to these clusters and indicate the potential future of the cluster forming in SDC335.

Use of future high resolution spectral line and dust continuum observations of SDC335 would lead to more precise knowledge of accretion rates of each object and detection of the mass fragmentation and, indeed segregation. These would provide more details of how SDC335 is forming a stellar cluster similar to the Trapezium cluster and NGC 6334 I(N).

Acknowledgements. The Australia Telescope Compact Array is part of the Australia Telescope which is funded by the Commonwealth of Australia for operation as a National Facility managed by CSIRO. This paper makes use of the following ALMA data: ADS/JAO.ALMA\#2011.0.00474.S. ALMA is a partnership of ESO (representing its member states), NSF (USA) and NINS (Japan), together with NRC (Canada) and NSC and ASIAA (Taiwan), in cooperation with the Republic of Chile. The Joint ALMA Observatory is operated by ESO, AUI/NRAO and NAOJ. The authors would like to thank the ATCA/ATNF staff who provided excellent help during the observations of the data this paper is based on. We also would like to thank the anonymous referee for their helpful comments on this paper. A.A. is funded by the STFC at the UK ARC Node. A.D.C. acknowledges funding from the European Research Council for 
the FP7 ERC starting grant project LOCALSTAR. A.T. is supported by a consolidated STFC grant to JBCA. J.E.P. is supported by the Swiss National Science Foundation, project number CRSII2_141880.

\section{References}

Bartkiewicz, A., Szymczak, M., van Langevelde, H. J., Richards, A. M. S., \& Pihlström, Y. M. 2009, A\&A, 502, 155

Beckwith, S. V. W., Sargent, A. I., Chini, R. S., \& Guesten, R. 1990, AJ, 99, 924 Beuther, H., Henning, T., Linz, H., et al. 2010, A\&A, 518, L78

Breen, S. L., Caswell, J. L., Ellingsen, S. P., \& Phillips, C. J. 2010, MNRAS, 406, 1487

Breen, S. L., Ellingsen, S. P., Contreras, Y., et al. 2013, MNRAS, 435, 524

Caswell, J. L., Fuller, G. A., Green, J. A., et al. 2011, MNRAS, 417, 1964

Churchwell, E., Sievers, A., \& Thum, C. 2010, A\&A, 513, A9

Cyganowski, C. J., Whitney, B. A., Holden, E., et al. 2008, AJ, 136, 2391

Cyganowski, C. J., Brogan, C. L., Hunter, T. R., \& Churchwell, E. 2009, ApJ, 702,1615

Davies, B., Hoare, M. G., Lumsden, S. L., et al. 2011, MNRAS, 416, 972

Duarte-Cabral, A., Bontemps, S., Motte, F., et al. 2013, A\&A, 558, A125

Fish, V. L. 2007, in IAU Symp. 242, eds. J. M. Chapman, \& W. A. Baan, 71

Fuller, G. A., Williams, S. J., \& Sridharan, T. K. 2005, A\&A, 442, 949

Garay, G., Brooks, K. J., Mardones, D., Norris, R. P., \& Burton, M. G. 2002, ApJ, 579, 678

Griffin, M. J., Abergel, A., Abreu, A., et al. 2010, A\&A, 518, L3

Hildebrand, R. H. 1983, QJRAS, 24, 267

Hillenbrand, L. A. 1997, AJ, 113, 1733

Hillenbrand, L. A., \& Hartmann, L. W. 1998, ApJ, 492, 540

Hoare, M. G., Lumsden, S. L., Oudmaijer, R. D., et al. 2004, in Milky Way

Surveys: The Structure and Evolution of our Galaxy, eds. D. Clemens, R. Shah, \& T. Brainerd, ASP Conf. Ser., 317, 156

Hosokawa, T., \& Omukai, K. 2009, ApJ, 691, 823

Hosokawa, T., Yorke, H. W., \& Omukai, K. 2010, ApJ, 721, 478

Hunter, T. R., Brogan, C. L., Cyganowski, C. J., \& Young, K. H. 2014, ApJ, 788, 187
Klaassen, P. D., Testi, L., \& Beuther, H. 2012, A\&A, 538, A140

Kroupa, P. 2002, Science, 295, 82

Kurtz, S. 2005, in Massive Star Birth: A Crossroads of Astrophysics, eds. R. Cesaroni, M. Felli, E. Churchwell, \& M. Walmsley, IAU Symp., 227, 111 Kurtz, S., Hofner, P., \& Álvarez, C. V. 2004, ApJS, 155, 149

Lada, C. J., \& Lada, E. A. 2003, ARA\&A, 41, 57

McKee, C. F., \& Tan, J. C. 2003, ApJ, 585, 850

McMullin, J. P., Waters, B., Schiebel, D., Young, W., \& Golap, K. 2007, in Astronomical Data Analysis Software and Systems XVI, eds. R. A. Shaw, F. Hill, \& D. J. Bell, ASP Conf. Ser., 376, 127

Mezger, P. G., \& Henderson, A. P. 1967, ApJ, 147, 471

Minier, V., Ellingsen, S. P., Norris, R. P., \& Booth, R. S. 2003, A\&A, 403, 1095

Molinari, S., Swinyard, B., Bally, J., et al. 2010, PASP, 122, 314

Mottram, J. C., Hoare, M. G., Davies, B., et al. 2011, ApJ, 730, L33

Muench, A. A., Lada, E. A., Lada, C. J., \& Alves, J. 2002, ApJ, 573, 366

Peretto, N., \& Fuller, G. A. 2009, A\&A, 505, 405

Peretto, N., Fuller, G. A., Duarte-Cabral, A., et al. 2013, A\&A, 555, A112

Pilbratt, G. L., Riedinger, J. R., Passvogel, T., et al. 2010, A\&A, 518, L1

Poglitsch, A., Waelkens, C., Geis, N., et al. 2010, A\&A, 518, L2

Rathborne, J. M., Jackson, J. M., Zhang, Q., \& Simon, R. 2008, ApJ, 689, 1141

Rathborne, J. M., Jackson, J. M., Chambers, E. T., et al. 2010, ApJ, 715, 310

Reid, M. J., Menten, K. M., Zheng, X. W., et al. 2009, ApJ, 700, 137

Reynolds, S. P. 1986, ApJ, 304, 713

Rygl, K. L. J., Wyrowski, F., Schuller, F., \& Menten, K. M. 2013, A\&A, 549, A5 Salpeter, E. E. 1955, ApJ, 121, 161

Sánchez-Monge, Á., Pandian, J. D., \& Kurtz, S. 2011, ApJ, 739, L9

Schuller, F., Menten, K. M., Contreras, Y., et al. 2009, A\&A, 504, 415

Shu, F. H. 1977, ApJ, 214, 488

Spitzer, L. 1978, Physical Processes in the Interstellar Medium, 1st edn. (John Wiley \& Sons, Inc)

Traficante, A., Fuller, G. A., Pineda, J. E., \& Pezzuto, S. 2015, A\&A, 574, A119

Wilson, T. L., Boboltz, D. A., Gaume, R. A., \& Megeath, S. T. 2003, ApJ, 597, 434

Wilson, W. E., Ferris, R. H., Axtens, P., et al. 2011, MNRAS, 416, 832

Xu, Y., Li, J. J., Hachisuka, K., et al. 2008, A\&A, 485, 729

Zhu, L., Zhao, J.-H., Wright, M. C. H., et al. 2013, ApJ, 779, 51 